\title{
Conservative tidal effects in compact binary systems to next-to-leading post-Minkowskian order
}

\author{
Gregor Kälin $\oplus^{1,2, *}$ Zhengwen Liu $\odot^{2, \dagger}$ and Rafael A. Porto $\oplus^{2, \$}$ \\ ${ }^{1}$ SLAC National Accelerator Laboratory, Stanford University, \\ Stanford, California 94309, USA \\ ${ }^{2}$ Deutsches Elektronen-Synchrotron DESY, Notkestrasse 85, \\ 22607 Hamburg, Germany
}

(Received 17 August 2020; accepted 27 October 2020; published 9 December 2020)

\begin{abstract}
Using the Effective Field Theory approach together with the boundary-to-bound map, we compute the next-to-leading order (NLO) post-Minkowskian (PM) tidal effects in the conservative dynamics of compact binary systems. We derive the mass and current quadrupole and, for the first time, octupole corrections to the binding energy for circular orbits at $\mathcal{O}\left(G^{3}\right)$. Our results are consistent with the test-body limit as well as the existent post-Newtonian literature. We also reconstruct a Hamiltonian incorporating tidal effects to NLO in the PM expansion and find complete agreement with the recent derivation of its quadrupolar part using the classical limit of scattering amplitudes.
\end{abstract}

DOI: $10.1103 /$ PhysRevD.102.124025

\section{INTRODUCTION}

The demonstrated feasibility of direct detection of gravitational waves (GWs) from binary systems [1,2], and, in particular, the observation of neutron star inspirals [3], has revealed a new window to explore compact objects in an unprecedented fashion [4-6]. Not only do GWs carry the imprint of the equation of state of nuclear matter through tidal effects [7-9], but they have also opened new frontiers for beyond the standard model searches [10-12], as well as the exploration of the remarkable properties of black holes in Einstein's gravity $[5,6]$. On the other hand, distinguishing the properties of compact objects from tidal disruptions is a daunting task requiring a high level of analytic control, to at least fifth post-Newtonian (5PN) order [5,6], while lifting several degeneracies may also require an even a higher level of precision for waveform modeling.

The Effective Field Theory (EFT) formalism for PN sources introduced in [13], which has already achieved a high level of analytic accuracy both for nonspinning

\footnotetext{
*gregor.kaelin@desy.de zhengwen.liu@desy.de *rafael.porto@desy.de
}

Published by the American Physical Society under the terms of the Creative Commons Attribution 4.0 International license. Further distribution of this work must maintain attribution to the author(s) and the published article's title, journal citation, and DOI. Funded by SCOAP ${ }^{3}$.
[14-28] and spinning binaries [29-42], is tailor made to incorporate finite-size effects; see, e.g., [13,43-47]. For instance, it was used in [48] to obtain the next-to-next-toleading-order (NNLO) contributions to the equations of motion to 7PN order. However, partially due to the repurposing of powerful tools from the amplitudes program, e.g., [49-58], as well as EFT, e.g., [49,50], the boundary-to-bound dictionary $[59,60]$ and other developments, e.g., [61-65], it has become apparent that the study of scattering in the post-Minkowskian (PM) expansion may ultimately push further the frontiers of analytic understanding of binary systems. With these ideas at hand, a worldline EFT approach was developed in [66] and readily implemented in [67] to reach the present state-of-the-art at 3PM $[52,53,56]$. Our purpose here is to extend the calculation of leading tidal effects in [66] (see also, [65,68]) and compute the mass and current quadrupolar and octupolar tidal effects to NLO in the PM expansion. While the latter are presented for the first time, we find agreement for the former with the recent results in [69]. The derivation in [69] uses the classical limit of the scattering amplitude augmented with higherderivative interactions and standard Feynman diagrams, together with the impetus formula [59]. Although Feynman's tools are also at the core of our approach, the formalisms are rather different. In particular, unlike the derivations in $[52,53,56,69]$, ours is reduced to (massless) integrals, whose velocity dependence can be obtained via differential equation from the EFT with static classical sources [67], which greatly simplifies the calculations. 


\section{A. Extended objects in the EFT approach}

Following [13], tidal effects are incorporated in [66] by including a series of higher-derivative terms in the worldline action, ${ }^{1,2}$

$$
\begin{aligned}
S_{\mathrm{pp}}= & \sum_{a=1,2} \int \mathrm{d} \tau_{a}\left(-\frac{m_{a}}{2} g_{\mu \nu} v_{a}^{\mu} v_{a}^{\nu}+c_{E^{2}}^{(a)} E_{\mu \nu} E^{\mu \nu}\right. \\
& \left.+c_{B^{2}}^{(a)} B_{\mu \nu} B^{\mu \nu}-c_{\tilde{E}^{2}}^{(a)} E_{\mu \nu \alpha} E^{\mu \nu \alpha}-c_{\tilde{B}^{2}}^{(a)} B_{\mu \nu \alpha} B^{\mu \nu \alpha}+\cdots\right),
\end{aligned}
$$

with $v_{a}^{\mu}$ each particle's velocity, $\left(c_{E^{2}}^{(a)}, c_{B^{2}}^{(a)}\right)$ and $\left(c_{\tilde{E}^{2}}^{(a)}, c_{\tilde{B}^{2}}^{(a)}\right)$ the mass and current quadrupole and octupole tidal Love numbers, respectively. The couplings are written in terms of the electric- and magnetic-type components of the Riemann (Weyl) tensor and its dual,

$$
\begin{aligned}
E_{\alpha \beta} & =R_{\mu \alpha \nu \beta} v^{\mu} v^{\nu}, \quad B_{\alpha \beta}=R_{\mu \alpha \nu \beta}^{\star} v^{\mu} v^{\nu}, \\
E_{\alpha \beta \gamma} & =\nabla_{\{\alpha}^{\perp} R_{\beta \rho \gamma\} \nu} v^{\rho} v^{\nu}, \quad B_{\alpha \beta \gamma}=\nabla_{\{\alpha}^{\perp} R_{\beta \gamma \gamma\} \nu}^{\star} v^{\rho} v^{\nu},
\end{aligned}
$$

where $\nabla_{\alpha}^{\perp}$ is the covariant derivative projected orthogonal to the velocity, and $\{\ldots\}$ stands for symmetrization. The two-body effective action is obtained by integrating out the metric field in the weak-field and saddle-point approximation via Feynman diagrams $[66,67]$. We use the convention $\eta_{\mu \nu}=\operatorname{diag}(+,-,-,-)$ for the Minkowski metric. Intermediate divergences are handled by dimensional regularization in $D=4-2 \epsilon$ dimensions.

\section{B. Scattering angle}

In the EFT formalism of [66], the scattering angle is computed via the impulse. The latter follows iteratively from the effective Lagrangian,

$$
\Delta p_{a}^{\mu}=-\eta^{\mu \nu} \int_{-\infty}^{+\infty} \mathrm{d} \tau_{a} \frac{\partial \mathcal{L}_{\text {eff }}}{\partial x_{a}^{\nu}}\left(x_{a}\left(\tau_{a}\right)\right)
$$

by inputting the PM expansion of the trajectories,

$$
x_{a}^{\mu}\left(\tau_{a}\right)=b_{a}^{\mu}+u_{a}^{\mu} \tau_{a}+\sum_{n} \delta^{(n)} x_{a}^{\mu}\left(\tau_{a}\right),
$$

\footnotetext{
${ }^{1}$ The action in Eq. (1) is equivalent to the reparameterizationinvariant one in [13], up to higher orders in the curvature. In the presence of finite-size terms, the gauge choice $e_{a}=1$ for the einbein sets $\tau_{a}$ as the proper time at future and past infinity. The relative signs are due to our flat-metric convention.

${ }^{2}$ In principle, there are other operators, involving time derivatives of the Riemann tensor, which are relevant in the PM expansion (see [65]). In fact, due to the vanishing of Love numbers [70-72], these represent the first nonzero corrections beyond minimal coupling for (nonrotating) black holes. However, for neutron stars, we expect these conservative (absorptive) effects to be suppressed with respect to higher order multipoles. For instance, time-dependent terms were not included in [71]. In principle one can include higher dimensional operators also in the Einstein-Hilbert action, see e.g., [73-75].
}

with $b^{\mu} \equiv b_{1}^{\mu}-b_{2}^{\mu}$ the impact parameter and $u_{a}$ the incoming velocities. The leading $\mathcal{L}_{\text {eff }}$ also contributes to NLO when evaluated on (4). We refer to these corrections as iterations $[66,67]$. The deflection angle is given by

$$
2 \sin \frac{\chi}{2}=\chi+\mathcal{O}\left(\chi^{3}\right)=\frac{\sqrt{-\Delta p_{a}^{2}}}{p_{\infty}},
$$

where $\quad p_{\infty}=\mu \frac{\sqrt{\gamma^{2}-1}}{\Gamma}, \quad$ with $\quad \Gamma \equiv \frac{E}{M}=\sqrt{1+2 \nu(\gamma-1)}$, $(M, E)$ the total mass/energy, $\mu=m_{1} m_{2} / M$ the reduced mass, and $\nu=\mu / M$ the symmetric mass ratio. Throughout this paper, we use the notation

$$
\gamma \equiv u_{1} \cdot u_{2}=\frac{p_{1} \cdot p_{2}}{m_{1} m_{2}}=1+\mathcal{E}+\frac{\nu}{2} \mathcal{E}^{2},
$$

where $\mathcal{E}=(E-M) / \mu$ is the (reduced) binding energy.

\section{Feynman master integrals}

At leading PM order, only the diagram in Fig. 1(b) contributes. The derivation for the quadrupole coupling was carried out in [66] and can be easily extended to the octupole term. For the NLO effects, the remaining diagrams in Fig. 1 are needed, including Fig. 1(a), which is required to compute the iterations. As discussed in $[66,67]$, in addition to the standard massless $1 / k^{2}$ propagators for the gravitational field, we have linear ones, $\left(k \cdot u_{a} \mp i 0\right)^{-1}$, which arise from the expansion in Eq. (4). We restrict ourselves to the impulse in the direction of the impact parameter, which is sufficient to obtain the scattering angle [66]. As in [67], the computation is reduced to terms involving the (transverse) Fourier transform in the transfer momentum of a series of two loop (cut) integrals.

As it turns out, a subset of the family of master integrals in [67] is sufficient to compute all of the diagrams in Fig. 1, including the iterations. As it was discussed in [67], the $\gamma$ dependence is obtained either by going to the rest frame of one of the particles or through differential equations whose boundary conditions are extracted from the static limit. In all cases, the integrals are reduced to the same type that appear in the computation of tidal effects in the EFT with PN sources [15]. The intermediate divergences either cancel out or yield contact terms that do not enter in the classical limit. Hence, we do not encounter ultraviolet poles requiring a counterterm contribution in the effective action

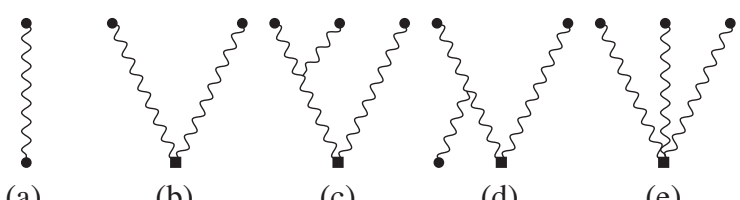

(a)

(b)

(c)

(d)

(e)

FIG. 1. Feynman diagrams needed for tidal effects to NLO. The square represents the finite-size couplings in Eq. (1). 
in Eq. (1) at this order. Not surprisingly, at the end of the day, the resulting tidal effects also feature the (in)famous factor of $\sinh ^{-1} \sqrt{(\gamma-1) / 2}$, first observed in the monopole contributions at NNLO in [52,53] and later confirmed in $[58,67]$.

\section{Scattering data}

Garnering all the ingredients for the impulse projected in the direction of the impact parameter, the scattering angle then follows from Eq. (5), yielding for the quadrupolar tidal effects [with $\left.1 / j \equiv G M \mu /\left(p_{\infty} b\right)\right]$

$$
\begin{aligned}
\frac{\Delta \chi_{(E, B)}}{\Gamma}= & \frac{45 \pi}{64} \frac{\left(\gamma^{2}-1\right)^{2}}{(\Gamma j)^{6}}\left[\left(35 \gamma^{4}-30 \gamma^{2}-5\right) \lambda_{B^{2}}+\left(35 \gamma^{4}-30 \gamma^{2}+11\right) \lambda_{E^{2}}\right] \\
& +\frac{192}{35} \frac{\left(\gamma^{2}-1\right)^{3 / 2}}{(\Gamma j)^{7}}\left[\left(160 \gamma^{6}-192 \gamma^{4}+30 \gamma^{2}+2\right) \lambda_{B^{2}}+\left(160 \gamma^{6}-192 \gamma^{4}+72 \gamma^{2}-5\right) \lambda_{E^{2}}\right] \\
& +\frac{96 \nu}{35} \frac{\sqrt{\gamma^{2}-1}}{(\Gamma j)^{7}} \kappa_{B^{2}}\left[224 \gamma^{9}-320 \gamma^{8}-728 \gamma^{7}+704 \gamma^{6}+5488 \gamma^{5}-444 \gamma^{4}+66262 \gamma^{3}+56 \gamma^{2}+28084 \gamma+4\right] \\
& +\frac{96 \nu}{35} \frac{\sqrt{\gamma^{2}-1}}{(\Gamma j)^{7}} \kappa_{E^{2}}\left[224 \gamma^{9}-320 \gamma^{8}-728 \gamma^{7}+704 \gamma^{6}+5628 \gamma^{5}-528 \gamma^{4}+65982 \gamma^{3}+154 \gamma^{2}+28329 \gamma-10\right] \\
& -\frac{576 \nu \sqrt{\gamma^{2}-1}}{(\Gamma j)^{7}}\left[\left(440 \gamma^{4}+474 \gamma^{2}+32\right) \kappa_{B^{2}}+\left(440 \gamma^{4}+474 \gamma^{2}+33\right) \kappa_{E^{2}}\right] a_{\operatorname{sh}(\gamma)},
\end{aligned}
$$

where we used the shortened notation $a_{\mathrm{sh}(\gamma)} \equiv\left(\gamma^{2}-1\right)^{-1 / 2} \sinh ^{-1} \sqrt{(\gamma-1) / 2}$; whereas for the octupolar contribution,

$$
\begin{aligned}
\frac{\Delta \chi_{(\tilde{E}, \tilde{B})}=}{\Gamma}= & \frac{525 \pi}{512(\Gamma j)^{8}}\left(\gamma^{2}-1\right)^{3}\left[\left(21 \gamma^{6}+385 \gamma^{4}-305 \gamma^{2}+91\right) \lambda_{\tilde{E}^{2}}+\left(21 \gamma^{6}+385 \gamma^{4}-385 \gamma^{2}-21\right) \lambda_{\tilde{B}^{2}}\right] \\
& +\frac{512\left(\gamma^{2}-1\right)^{5 / 2}}{3003(\Gamma j)^{9}}\left[\left(4800 \gamma^{8}+77520 \gamma^{6}-74888 \gamma^{4}+17707 \gamma^{2}+1888\right) \lambda_{\tilde{E}^{2}}\right. \\
& \left.+\left(4800 \gamma^{8}+77520 \gamma^{6}-87472 \gamma^{4}+5552 \gamma^{2}-400\right) \lambda_{\tilde{B}^{2}}\right] \\
& +\frac{128 \nu \sqrt{\gamma^{2}-1} \kappa_{\tilde{B}^{2}}}{3003(\Gamma j)^{9}}\left[27456 \gamma^{13}-19200 \gamma^{12}+205920 \gamma^{11}-271680 \gamma^{10}-1589016 \gamma^{9}+950848 \gamma^{8}+22048884 \gamma^{7}\right. \\
& \left.-1032064 \gamma^{6}+579540390 \gamma^{5}+395904 \gamma^{4}+826613931 \gamma^{3}-25408 \gamma^{2}+148331040 \gamma+1600\right] \\
& +\frac{128 \nu \sqrt{\gamma^{2}-1} \kappa_{\tilde{E}^{2}}}{3003(\Gamma j)^{9}}\left[27456 \gamma^{13}-19200 \gamma^{12}+205920 \gamma^{11}-271680 \gamma^{10}-1468896 \gamma^{9}+900512 \gamma^{8}+21724560 \gamma^{7}\right. \\
& \left.-980012 \gamma^{6}+580453302 \gamma^{5}+433656 \gamma^{4}+837773079 \gamma^{3}-55724 \gamma^{2}+155291994 \gamma-7552\right] \\
& -\frac{3840 \nu \sqrt{\gamma^{2}-1}}{(\Gamma j)^{9}}\left[\left(7292 \gamma^{6}+19484 \gamma^{4}+7905 \gamma^{2}+288\right) \kappa_{\tilde{B}^{2}}+\left(7292 \gamma^{6}+19644 \gamma^{4}+8141 \gamma^{2}+310\right) \kappa_{\tilde{E}^{2}}\right] a_{\operatorname{sh}(\gamma)} .
\end{aligned}
$$

In these expressions, we introduced the parameters

$$
\begin{aligned}
& \lambda_{E^{2}} \equiv \frac{1}{G^{4} M^{5}}\left(m_{2} \frac{c_{E^{2}}^{(1)}}{m_{1}}+m_{1} \frac{c_{E^{2}}^{(2)}}{m_{2}}\right), \\
& \kappa_{E^{2}} \equiv \lambda_{E^{2}}+\frac{c_{E^{2}}+c_{E^{2}}^{(2)}}{G^{4} M^{5}}=\frac{1}{G^{4} M^{4}}\left(\frac{c_{E^{2}}^{(1)}}{m_{1}}+\frac{c_{E^{2}}^{(2)}}{m_{2}}\right),
\end{aligned}
$$

and similarly for all the other couplings, normalized with $1 / G^{6} M^{7}$ for the octupole Love numbers. We find the expression in Eq. (7) to be fully equivalent to Eq. (13) in [69]. Notice that, as it happens also at leading PM order $[65,66]$, the electric- and magnetic-type tidal effects have a strikingly similar behavior in the high-energy limit. (Likewise, this is encoded in the rather simple factor in the $\lambda_{1}$ coupling to the Kretschmann scalar in [69].)

\section{E. Probe limit}

A nontrivial test for our results is the consistency with the test-particle limit. We computed the scattering angle for 
a tidally deformed object by means of the on-shell condition in a Schwarzschild background,

$$
\begin{aligned}
g_{\mathrm{Sch}}^{\mu \nu} p_{\mu}^{(a)} p_{\nu}^{(a)}= & m_{a}^{2}-2 m_{a}\left(c_{E^{2}}^{(a)}\left(E_{\mu \nu}^{\mathrm{Sch}}\right)^{2}+c_{B^{2}}^{(a)}\left(B_{\mu \nu}^{\mathrm{Sch}}\right)^{2}\right. \\
& \left.-c_{\tilde{E}^{2}}^{(a)}\left(E_{\alpha \mu \nu}^{\mathrm{Sch}}\right)^{2}-c_{\tilde{B}^{2}}^{(a)}\left(B_{\alpha \mu \nu}^{\mathrm{Sch}}\right)^{2}\right) .
\end{aligned}
$$

We constructed the radial action for hyperbolic motion, from which we derived the scattering angle in the PM expansion via differentiation. Identifying the incoming (reduced) energy with the boost factor $\left(\mathcal{E}_{0} \rightarrow \gamma\right)$, we found that the expressions in Eqs. (7) and (8) are consistent with the deflection of a tidally disrupted test body in a black hole background. Not surprisingly, the probe limit also fixes the leading tidal effects [59]. See the Appendix for more details.

\section{F. Boundary-to-Bound dictionary}

The (reduced) radial action [59,60],

$$
i_{r}=\frac{p_{\infty}}{\sqrt{-p_{\infty}^{2}}} \chi_{j}^{(1)}-j\left(1+\frac{2}{\pi} \sum_{n=1}^{\infty} \frac{\chi_{j}^{(2 n)}}{(1-2 n) j^{2 n}}\right),
$$

is built from the PM expansion of the deflection angle,

$$
\frac{\chi}{2}=\sum_{n} \chi_{b}^{(n)}\left(\frac{G M}{b}\right)^{n}=\sum_{n} \frac{\chi_{j}^{(n)}}{j^{n}},
$$

via analytic continuation in the binding energy. Similarly to what occurs at 3PM with the monopole term [59,60,67], we can incorporate the information in the NLO tidal effects by performing a PN truncation. To do so, we use the map in [59] to write the $P_{n} \mathrm{~s}$ in the expansion of the square of the center-of-mass momentum for each particle,

$$
p^{2}=p_{\infty}^{2}+\sum_{n=1}^{\infty} P_{n}(E)\left(\frac{G}{r}\right)^{n}
$$

as a function of the PM coefficients in (12). This allows us to read off the finite-size contributions to $\left\{P_{6}, P_{7}, P_{8}, P_{9}\right\}$ from Eqs. (7) and (8). We then use the inverse map [59], e.g.,

$$
\begin{aligned}
\chi_{j}^{(8)}= & \frac{105 \pi}{64}\left(\frac{\bar{P}_{2}^{4}}{12}+\frac{\bar{P}_{1}^{2} \bar{P}_{3}^{2}}{2}+\bar{P}_{1} \bar{P}_{2}^{2} \bar{P}_{3}+\hat{p}_{\infty}^{2}\left(\bar{P}_{1}^{2} \bar{P}_{6}\right.\right. \\
& \left.\left.+P_{2} P_{3}^{2}\right)+\hat{p}_{\infty}^{4}\left(\bar{P}_{1} \bar{P}_{7}+\bar{P}_{2} \bar{P}_{6}\right)+\frac{\hat{p}_{\infty}^{6}}{3} \bar{P}_{8}+\cdots\right),
\end{aligned}
$$

$$
\begin{aligned}
\chi_{j}^{(10)}= & \frac{315 \pi}{512}\left(\frac{\bar{P}_{2}^{5}}{5}+\bar{P}_{1}^{4} \bar{P}_{6}+\cdots+4 \hat{p}_{\infty}^{2}\left(3 \bar{P}_{1}^{2} \bar{P}_{2} \bar{P}_{6}\right.\right. \\
& \left.+\bar{P}_{1}^{3} \bar{P}_{7}+\cdots\right)+6 \hat{p}_{\infty}^{4}\left(\bar{P}_{1}^{2} \bar{P}_{8}+2 \bar{P}_{1} \bar{P}_{2} \bar{P}_{7}+\cdots\right) \\
& \left.+4 \hat{p}_{\infty}^{6}\left(\bar{P}_{2} \bar{P}_{8}+\bar{P}_{1} \bar{P}_{9}+\bar{P}_{3} \bar{P}_{7}\right)+\hat{p}_{\infty}^{8} \bar{P}_{10}+\cdots\right),
\end{aligned}
$$

with $\bar{P}_{n} \equiv P_{n} /\left(\mu^{2} M^{n}\right)$ and $\hat{p}_{\infty}=p_{\infty} / \mu$, to input the known information into the $\chi_{j}^{(2 n)}$, s in (11). We have displayed only a subset of the relevant coefficients and their respective dependence on the $\left\{P_{1}, P_{2}, P_{3}\right\}$ at $3 \mathrm{PM}$, as well as the $\left\{P_{6}, P_{7}, P_{8}, P_{9}\right\}$ whose tidal contributions we have computed. Notice we are still missing the quadrupole corrections to $\left\{P_{8}, P_{9}\right\}$, as well as $P_{n \geq 10}$. However, the reader will immediately notice the factors of $\hat{p}_{\infty}^{2}$ attached to each term in Eqs. (14) and (15) (depending on the number of $P_{n} \mathrm{~s}$ involved) [59,60]. After analytic continuation, the $\hat{p}_{\infty}^{2}$ scales with the (reduced) binding energy of the binary. Hence, since the static limit of (13) is well defined, we can consistently truncate Eq. (11) by ignoring terms that enter at higher PN orders. There is still one subtlety left. While the analytic continuation formally maps the $1 / j$ expansion of the observables between hyperbolic and elliptic motion, for the latter case, we have the additional PN scaling $j^{-1} \simeq\left|\hat{p}_{\infty}\right|$, which mixes the power counting. Therefore, we have to retain also higher orders in the $1 / j$ expansion of (11). For instance, by keeping the $\left\{P_{1}, P_{6}\right\}$ contributions to the deflection angle in Eqs. (14) and (15), we recover the exact value of the periastron advance in the Newtonian limit in [65]. Different powers in $1 / j$ are also necessary to match the PN results for the monopole terms at higher PM/PN orders $[59,60]$.

The procedure is now straightforward, allowing us to derive gauge-invariant observables directly from the analytically continued radial action $[59,60,66,67]$. For instance, we readily obtain the azimuthal orbital frequency, $\Omega_{\phi}(j, \gamma)$, by taking derivatives of Eq. (11), with respect to the binding energy and angular momentum. For the case of circular orbits, we proceed as follows $[59,60,66]$. First, we solve for $j_{\text {circ }}(\gamma)$ with the condition $i_{r}=0$ in (11), including also the 3PM monopole corrections [59,60,66,67]. We plug it back into $\Omega_{\phi}\left(j_{\text {circ }}(\gamma), \gamma\right) \equiv \Omega_{\text {circ }}(\gamma)$, which can then be inverted to extract the binding energy as a function of the orbital frequency. (Alternatively, we have checked that the first law of binary dynamics [76] holds for tidal effects.) Bundling the terms together and keeping up to $2 \mathrm{PN}$ corrections in each sector, we find 


$$
\begin{aligned}
\Delta \mathcal{E}_{\mathrm{T}}= & x\left[18 \lambda_{E^{2}} x^{5}+11\left(3(1-\nu) \lambda_{E^{2}}+6 \lambda_{B^{2}}+5 \nu \kappa_{E^{2}}\right) x^{6}+\left(390 \lambda_{\tilde{E}^{2}}-\frac{13}{28}\left(161 \nu^{2}-161 \nu-132\right) \lambda_{E^{2}}-\frac{1326 \nu}{7} \kappa_{B^{2}}\right.\right. \\
& \left.+\frac{13}{28}(616 \nu+699) \lambda_{B^{2}}+\frac{13 \nu}{84}(490 \nu-729) \kappa_{E^{2}}+\frac{13}{6} \Delta \bar{P}_{8, \mathrm{stc}}^{(E, B)}\right) x^{7}+75\left(45 \nu \kappa_{\tilde{E}^{2}}-(13 \nu+3) \lambda_{\tilde{E}^{2}}+16 \lambda_{\tilde{B}^{2}}\right) x^{8} \\
& \left.-\left(\frac{85}{36}\left(1083 \nu^{2}+1539 \nu+163\right) \lambda_{\tilde{E}^{2}}+\frac{27200 \nu}{3} \kappa_{\tilde{B}^{2}}-\frac{85}{4}(270 \nu+383) \nu \kappa_{\tilde{E}^{2}}-\frac{680}{9}(90 \nu+173) \lambda_{\tilde{B}^{2}}-\frac{17}{6} \Delta \bar{P}_{10, \mathrm{stc}}^{(\tilde{E}, \tilde{B})}\right) x^{9}\right]
\end{aligned}
$$

with $x \equiv\left(G M \Omega_{\text {circ }}\right)^{2 / 3}$ the standard PN parameter. The $\Delta \bar{P}_{8, \text { stc }}^{(E, B)}$ and $\Delta \bar{P}_{10, \text { stc }}^{(\widetilde{E}, \tilde{B})}$ are the contributions in the static limit $(\gamma \rightarrow 1)$ from the quadrupole and octupole couplings at NNLO in $G$, respectively. The expression in Eq. (16) agrees with the results in Eq. 6.5b of [48] for the quadrupole couplings to $6 \mathrm{PN}$, as well as the leading octupole at 7PN. Moreover, the difference at 7PN in [48] is only due to the static limit of $P_{8}$. We can extract its value using Eq. (14) with the $\chi_{j}^{(8)}$, which follows from the Lagrangian to 7PN obtained in [48], yielding

$$
\begin{aligned}
\Delta \bar{P}_{8, \mathrm{stc}}^{(E, B)}= & \frac{1326}{7} \nu \kappa_{B^{2}}+(243-90 \nu) \nu \kappa_{E^{2}} \\
& +\left(45 \nu^{2}-\frac{885 \nu}{7}+\frac{675}{14}\right) \lambda_{E^{2}} \\
& -\left(234 \nu+\frac{837}{14}\right) \lambda_{B^{2}} .
\end{aligned}
$$

We have checked that its $\mathcal{O}\left(\nu^{0}\right)$ part is consistent with the probe limit (see the Appendix). The correction in Eq. (17) gives us the last ingredient for the binding energy at 7PN, while, at the same time, proves the equivalence of the derivations in [48] with a truncation of the PM results in the quadrupole sector to $\mathcal{O}\left(G^{3} v^{2}\right)$.

As advertised, the octupolar contributions at $8 \mathrm{PN}$ are presented for the first time. We also included the partial results at 9PN order, missing only the static corrections at NNLO in $G$ from $\Delta P_{10}$, whose $\mathcal{O}\left(\nu^{0}\right)$ part can be extracted from the probe limit (see Appendix),

$$
\Delta \bar{P}_{10, \mathrm{stc}}^{(\tilde{E}, \tilde{B})}=\frac{1}{3}\left(2050 \lambda_{\tilde{E}^{2}}-13120 \lambda_{\tilde{B}^{2}}\right)+\mathcal{O}(\nu) .
$$

\section{G. Hamiltonian}

The Boundary-to-Bound (B2B) map allows us to directly produce observables without a Hamiltonian. However, it is still instructive to reconstruct it using our dictionary [59]. We do so in the center-of-mass (isotropic) frame, where

$$
H\left(r, \boldsymbol{p}^{2}\right)=\sum_{n=0}^{\infty} \frac{c_{n}\left(\boldsymbol{p}^{2}\right)}{n !}\left(\frac{G}{r}\right)^{n},
$$

with $c_{0} \equiv \sum_{a} \sqrt{\boldsymbol{p}^{2}+m_{a}^{2}}$. The $c_{n}$ coefficients in Eq. (19) can be then obtained iteratively from the $P_{n} \mathrm{~s}$ in Eq. (13). The tidal contributions to the latter are collected in the Appendix, from which we derive the (lengthier) finite-size contributions to the former,

$$
\begin{aligned}
\Delta c_{6}= & -\frac{270 M^{7} \nu^{2}}{\Gamma^{2} \xi}\left[\left(35 \gamma^{4}-30 \gamma^{2}+11\right) \lambda_{E^{2}}+5\left(7 \gamma^{4}-6 \gamma^{2}-1\right) \lambda_{B^{2}}\right], \\
\Delta c_{7}= & \frac{270 M^{8} \nu^{2}}{\left(\gamma^{2}-1\right) \Gamma^{2} \xi}\left\{\frac{\nu}{\gamma^{2}-1}\left[\left(D_{7,1} \kappa_{E^{2}}+D_{7,2} \kappa_{B^{2}}\right)+\left(D_{7,3} \kappa_{E^{2}}+D_{7,4} \kappa_{B^{2}}\right) a_{\mathrm{sh}(\gamma)}\right]\right. \\
& +\left[D_{7,5}+\frac{\nu^{2}}{\Gamma^{7} \xi^{2}}\left(D_{7,7}+(\gamma-1) D_{7,9} \nu+(\gamma-1)^{3} D_{7,11} \nu^{2}\right)\right] \lambda_{E^{2}} \\
& \left.+\left[D_{7,6}+\frac{\left(\gamma^{2}-1\right) \nu^{2}}{\Gamma^{7} \xi^{2}}\left(D_{7,8}+(\gamma-1) D_{7,10} \nu+(\gamma-1)^{3} D_{7,12} \nu^{2}\right)\right] \lambda_{B^{2}}\right\}, \\
\Delta c_{8}= & -\frac{18900 M^{9} \nu^{2}}{\Gamma^{2} \xi}\left[\left(21 \gamma^{6}+385 \gamma^{4}-305 \gamma^{2}+91\right) \lambda_{\tilde{E}^{2}}+7\left(3 \gamma^{6}+55 \gamma^{4}-55 \gamma^{2}-3\right) \lambda_{\tilde{B}^{2}}\right],
\end{aligned}
$$




$$
\begin{aligned}
\Delta c_{9}= & \frac{302400 M^{10} \nu^{2}}{143\left(\gamma^{2}-1\right) \Gamma^{2} \xi}\left\{\frac{\nu}{\left(\gamma^{2}-1\right)^{2}}\left[\left(D_{9,1} \kappa_{\tilde{E}^{2}}+D_{9,2} \kappa_{\tilde{B}^{2}}\right)+\left(D_{9,3} \kappa_{\tilde{E}^{2}}+D_{9,4} \kappa_{\tilde{B}^{2}}\right) a_{\mathrm{sh}(\gamma)}\right]\right. \\
& +\left[D_{9,5}+\frac{1287 \nu^{2}}{16 \Gamma^{7} \xi^{2}}\left(D_{9,7}+(\gamma-1) D_{9,9} \nu+(\gamma-1)^{3} D_{9,11} \nu^{2}\right)\right] \lambda_{\tilde{E}^{2}} \\
& \left.+\left[D_{9,6}+\frac{1287 \nu^{2}\left(\gamma^{2}-1\right)}{16 \Gamma^{7} \xi^{2}}\left(D_{9,8}+(\gamma-1) D_{9,10} \nu+(\gamma-1)^{3} D_{9,12} \nu^{2}\right)\right] \lambda_{\tilde{B}^{2}}\right\} .
\end{aligned}
$$

The $D_{i, j}$ are polynomials in $\gamma$, which we display in the Appendix. We find agreement with the results for the quadrupolar contributions computed in [69], while the octupolar corrections beyond leading PN/PM order are derived here for the first time.

\section{CONCLUSION}

Motivated by probing compact objects via GW observations [4-12], we computed tidal effects in the conservative dynamics to NLO in the PM expansion. We used the EFT approach and B2B map developed in $[59,60,66,67]$ to calculate the mass and current quadrupolar and, for the first time, octupolar corrections to the scattering angle to NLO, from which we derived the binding energy for circular orbits. Ultimately, it is through the accurate reconstruction of finite-size effects that we will constrain the nature of compact objects, notably the one(s) recently observed in the so-called mass gap [77,78]. Measuring tidal responses is especially relevant for (nonrotating) black holes, due to their vanishing Love numbers [70-72] (see also, [79]), which offers a unique opportunity to search for new physics [5,11,12]. Our results thus provide new ingredients for accurate waveform modeling including tidal corrections.

Our derivation is also interesting with regards to the high-energy limit $(\gamma \rightarrow \infty)$. Remarkably, there is a pattern between the electric and magnetic quadrupolar as well as octupolar corrections, notably for the impulse at fixed impact parameter. For instance, the difference at leading PM order is $\mathcal{O}(1 / \gamma)$ in the quadrupole, as noted in [65], and $\mathcal{O}(\gamma)$ for the octupole. This feature extends to all orders in the probe limit, whereas, at $\mathcal{O}(\nu)$, the electric/magnetic split picks an extra factor of $\gamma$ for each multipole, except for the $a_{\mathrm{sh}(\gamma)}$. The mismatch in the impulse for the latter goes as $G^{3} \gamma^{-6(4)} \log \gamma$ for the quadrupole (octupole) coupling. We also find a softer behavior for the individual terms in comparison with the monopole, which instead scales as $G^{3} \gamma^{2} \log \gamma$ in the high-energy limit [67]. It would be interesting to understand these features and whether they persist at higher orders.

\section{ACKNOWLEDGMENTS}

R. A.P. is supported by the ERC-CoG "Precision Gravity: From the LHC to LISA," provided by the
European Research Council (ERC) under the European Union's H2020 research and innovation programme (Grant No. 817791). Z. L. and R. A. P. are supported by the DFG under Germany's Excellence Strategy Quantum Universe (No. 390833306). G. K. is supported by the Knut and Alice Wallenberg Foundation (Grant No. KAW 2018.0441) and, in part, by the U.S. DOE under Contract No. DE-AC0276SF00515. We also acknowledge extensive use of the xAct computer algebra packages [80].

\section{APPENDIX: SUPPLEMENTAL MATERIAL}

In this Appendix, we provide the details of the consistency check of our derivation of the scattering angle with the probe limit of a tidally-disrupted object in a Schwarzschild background. We also collect various fomulae which are relevant for the results presented in the main text.

Schwarzschild Background.-The consistency of our results with the test-particle limit can be shown directly in terms of the (gauge-invariant) scattering angle. Using the on-shell (constrain) condition due to the reparameterization invariance of the worldline effective action; or equivalently, using the relation between $p^{\mu}$ and $v^{\mu}$ and the normalization of the velocity which follows from the gauge choice $e_{a}=1$ for the einbein, we have

$$
\begin{aligned}
g_{\mathrm{Sch}}^{\mu \nu} p_{\mu}^{(a)} p_{\nu}^{(a)}= & m_{a}^{2}-2 m_{a}\left(c_{E^{2}}^{(a)}\left(E_{\mu \nu}^{\mathrm{Sch}}\right)^{2}+c_{B^{2}}^{(a)}\left(B_{\mu \nu}^{\mathrm{Sch}}\right)^{2}\right. \\
& \left.-c_{\tilde{E}^{2}}^{(a)}\left(E_{\alpha \mu \nu}^{\mathrm{Sch}}\right)^{2}-c_{\tilde{B}^{2}}^{(a)}\left(B_{\alpha \mu \nu}^{\mathrm{Sch}}\right)^{2}\right)+\cdots,
\end{aligned}
$$

up to terms quadratic in the tidal operators. We can then solve for $p_{r}^{(1)}$ as a function of the distance, the (reduced) energy, $\mathcal{E}_{0}^{(1)}$, angular momentum, $J_{0}^{(1)}$, and tidal Love numbers of the test body (which we take as particle 1). Afterwards, we construct the radial action, $\int p_{r}^{(1)} \mathrm{d} r$, such that the scattering angle follows via differentiation with respect to the angular momentum. Expanding in powers of $1 / j_{0} \equiv\left(G m_{2} m_{1}\right) / J_{0}^{(1)}$, replacing the energy by the boost factor, $\mathcal{E}_{0}^{(1)} \rightarrow \gamma$, and following the integration procedure described in, e.g., Ref. [65], we arrive at the following corrections due to tidal effects in the deflection angle in a Schwarzschild background, 


$$
\begin{aligned}
\Delta \chi_{(1)}^{\mathrm{Sch}}= & \frac{45 \pi}{64 j_{0}^{6}}\left(\gamma^{2}-1\right)^{2}\left(\left(35 \gamma^{4}-30 \gamma^{2}+11\right) \lambda_{E^{2}}^{(1)}+\left(35 \gamma^{4}-30 \gamma^{2}-5\right) \lambda_{B^{2}}^{(1)}\right) \\
& +\frac{192}{35 j_{0}^{7}}\left(\gamma^{2}-1\right)^{3 / 2}\left(\left(160 \gamma^{6}-192 \gamma^{4}+72 \gamma^{2}-5\right) \lambda_{E^{2}}^{(1)}+\left(160 \gamma^{6}-192 \gamma^{4}+30 \gamma^{2}+2\right) \lambda_{B^{2}}^{(1)}\right) \\
& +\frac{525 \pi}{512 j_{0}^{8}}\left(\gamma^{2}-1\right)^{3}\left(\left(21 \gamma^{6}+385 \gamma^{4}-305 \gamma^{2}+91\right) \lambda_{\tilde{E}^{2}}^{(1)}+\left(21 \gamma^{6}+385 \gamma^{4}-385 \gamma^{2}-21\right) \lambda_{\tilde{B}^{2}}^{(1)}\right) \\
& +\frac{63 \pi}{256 j_{0}^{8}}\left(\gamma^{2}-1\right)\left(\left(9009 \gamma^{8}-15246 \gamma^{6}+8484 \gamma^{4}-1666 \gamma^{2}+59\right) \lambda_{E^{2}}^{(1)}+21\left(\gamma^{2}-1\right)\left(429 \gamma^{6}-297 \gamma^{4}+27 \gamma^{2}+1\right) \lambda_{B^{2}}^{(1)}\right) \\
& +\frac{256}{77 j_{0}^{9}} \sqrt{\gamma^{2}-1\left(\left(14336 \gamma^{10}-32256 \gamma^{8}+25792 \gamma^{6}-8720 \gamma^{4}+1104 \gamma^{2}-25\right) \lambda_{E^{2}}^{(1)}\right.} \\
& \left.+\left(14336 \gamma^{10}-32256 \gamma^{8}+23680 \gamma^{6}-6080 \gamma^{4}+312 \gamma^{2}+8\right) \lambda_{B^{2}}^{(1)}\right) \\
& +\frac{512}{3003 j_{0}^{9}}\left(\gamma^{2}-1\right)^{5 / 2}\left(\left(4800 \gamma^{8}+77520 \gamma^{6}-74888 \gamma^{4}+17707 \gamma^{2}+1888\right) \lambda_{\tilde{E}^{2}}^{(1)}\right. \\
& \left.+\left(4800 \gamma^{8}+77520 \gamma^{6}-87472 \gamma^{4}+5552 \gamma^{2}-400\right) \lambda_{\tilde{B}^{2}}^{(1)}\right)+\mathcal{O}\left(1 / j_{0}^{10}\right)
\end{aligned}
$$

where $\quad \lambda_{E^{2}\left(B^{2}\right)}^{(1)} \equiv G^{-4} m_{2}^{-5}\left(m_{2} / m_{1}\right) c_{E^{2}\left(B^{2}\right)}^{(1)} \quad$ and $\quad \lambda_{\tilde{E}^{2}\left(\tilde{B}^{2}\right)}^{(1)} \equiv G^{-6} m_{2}^{-7}\left(m_{2} / m_{1}\right) c_{\tilde{E}^{2}\left(\tilde{B}^{2}\right)}^{(1)}$ The expression $\quad$ in $\quad($ A2), which to our knowledge is presented here for the first time, must be symmetrized to obtain the mirror image. The result neatly agrees with the test-body limit of the scattering angle shown in Eqs. (7) and (8). Moreover, using (A2) and the map from [59], we can also solve for the tidal correction to the momentum coefficients $\bar{P}_{n}^{\text {Sch }}$ in a Schwarzschild background, e.g.,

$$
\begin{aligned}
& \Delta \bar{P}_{6}^{\mathrm{Sch}}=\frac{3}{4}\left(35 \gamma^{4}-30 \gamma^{2}+11\right) \lambda_{E^{2}}^{(1)}+\frac{15}{4}\left(7 \gamma^{4}-6 \gamma^{2}-1\right) \lambda_{B^{2}}^{(1)}, \\
& \Delta \bar{P}_{7}^{\mathrm{Sch}}=\frac{3}{28}\left(110 \gamma^{4}+363 \gamma^{2}-305\right) \lambda_{E^{2}}^{(1)}+\frac{33}{28}\left(10 \gamma^{4}+33 \gamma^{2}+13\right) \lambda_{B^{2}}^{(1)}, \\
& \Delta \bar{P}_{8}^{\mathrm{Sch}}=\frac{9}{140}\left(544 \gamma^{4}-933 \gamma^{2}+1139\right) \lambda_{E^{2}}^{(1)}+\frac{9}{140}\left(544 \gamma^{4}-933 \gamma^{2}-541\right) \lambda_{B^{2}}^{(1)} \\
& +\frac{15}{16}\left(21 \gamma^{6}+385 \gamma^{4}-305 \gamma^{2}+91\right) \lambda_{\tilde{E}^{2}}^{(1)}+\frac{105}{16}\left(3 \gamma^{6}+55 \gamma^{4}-55 \gamma^{2}-3\right) \lambda_{\tilde{B}^{2}}^{(1)}, \\
& \Delta \bar{P}_{9}^{\mathrm{Sch}}=\frac{1}{880}\left(14302 \gamma^{4}+59187 \gamma^{2}-107149\right) \lambda_{E^{2}}^{(1)}+\frac{1}{880}\left(14302 \gamma^{4}+59187 \gamma^{2}+51251\right) \lambda_{B^{2}}^{(1)} \\
& -\frac{5}{6864}\left(71178 \gamma^{6}+1857639 \gamma^{4}-2313484 \gamma^{2}+940651\right) \lambda_{\tilde{E}^{2}}^{(1)} \\
& -\frac{5}{6864}\left(71178 \gamma^{6}+1857639 \gamma^{4}-2949548 \gamma^{2}-214789\right) \lambda_{\tilde{B}^{2}}^{(1)} \text {, } \\
& \Delta \bar{P}_{10}^{\mathrm{Sch}}=\frac{\left(42008 \gamma^{4}-108497 \gamma^{2}+257471\right)}{1540} \lambda_{E^{2}}^{(1)}+\frac{\left(42008 \gamma^{4}-108497 \gamma^{2}-123679\right)}{1540} \lambda_{B^{2}}^{(1)} \\
& +\frac{\left(872265 \gamma^{6}+37951761 \gamma^{4}-65175713 \gamma^{2}+34559887\right)}{12012} \lambda_{\tilde{E}^{2}}^{(1)} \\
& +\frac{\left(872265 \gamma^{6}+37951761 \gamma^{4}-83522041 \gamma^{2}-7834465\right)}{12012} \lambda_{\tilde{B}^{2}}^{(1)} \text {. }
\end{aligned}
$$

After adding the mirror images, the quadrupole contribution to $\bar{P}_{8}^{\mathrm{Sch}}(\gamma \rightarrow 1)$ exactly matches the static limit of the $\Delta P_{8}$ at $\mathcal{O}\left(\nu^{0}\right)$ shown in the main text. Similarly, the $\Delta P_{10}^{\mathrm{Sch}}(\gamma \rightarrow 1)$ yields the associated part of the full $\Delta P_{10}$ at $\mathcal{O}\left(\nu^{0}\right)$. Finally, notice that the probe limit also fixes the leading deflection for comparable masses. This is clear in impact parameter space, where the impulse remains the same and we only have to add the mirror image. Hence, replacing $p_{\infty}^{(1)} \rightarrow \mu \sqrt{\gamma^{2}-1} / \Gamma$ we obtain the two-body deflection angle at leading order. 
Momentum and Hamiltonian PM-coefficients.-The tidal corrections to the center-of-mass momentum,

$$
\Delta \mathbf{p}^{2}=\sum_{n=1}^{\infty} \Delta P_{n}(E)\left(\frac{G}{r}\right)^{n},
$$

are obtained from the map in [59]. Using the value of the NLO scattering angle reported in the main text, we find for the quadrupole contributions,

$$
\begin{aligned}
\Delta P_{6}= & \frac{3 M^{8} \nu^{2}}{4 \Gamma}\left(5\left(7 \gamma^{4}-6 \gamma^{2}-1\right) \lambda_{B^{2}}+\left(35 \gamma^{4}-30 \gamma^{2}+11\right) \lambda_{E^{2}}\right), \\
\Delta P_{7}= & \frac{3 M^{9} \nu^{2}}{28\left(\gamma^{2}-1\right) \Gamma}\left\{16\left(\left(160 \gamma^{6}-192 \gamma^{4}+30 \gamma^{2}+2\right) \lambda_{B^{2}}+\left(160 \gamma^{6}-192 \gamma^{4}+72 \gamma^{2}-5\right) \lambda_{E^{2}}\right)\right. \\
& -35 \Gamma\left(2 \gamma^{2}-1\right)\left(\left(35 \gamma^{4}-30 \gamma^{2}-5\right) \lambda_{B^{2}}+\left(35 \gamma^{4}-30 \gamma^{2}+11\right) \lambda_{E^{2}}\right) \\
& +\frac{8 \nu}{\gamma^{2}-1}\left[\left(224 \gamma^{9}-320 \gamma^{8}-728 \gamma^{7}+704 \gamma^{6}+5488 \gamma^{5}-444 \gamma^{4}+66262 \gamma^{3}+56 \gamma^{2}\right.\right. \\
& +28084 \gamma+4) \kappa_{B^{2}}+\left(224 \gamma^{9}-320 \gamma^{8}-728 \gamma^{7}+704 \gamma^{6}+5628 \gamma^{5}-528 \gamma^{4}+65982 \gamma^{3}+154 \gamma^{2}+28329 \gamma-10\right) \kappa_{E^{2}} \\
& \left.\left.-210\left(\left(440 \gamma^{4}+474 \gamma^{2}+32\right) \kappa_{B^{2}}+\left(440 \gamma^{4}+474 \gamma^{2}+33\right) \kappa_{E^{2}}\right) a_{\mathrm{sh}}(\gamma)\right]\right\},
\end{aligned}
$$

whereas for the octupolar corrections we have

$$
\begin{aligned}
\Delta P_{8}= & \frac{15 M^{10} \nu^{2}}{16 \Gamma}\left(\left(21 \gamma^{6}+385 \gamma^{4}-305 \gamma^{2}+91\right) \lambda_{\tilde{E}}^{2}+7\left(3 \gamma^{6}+55 \gamma^{4}-55 \gamma^{2}-3\right) \lambda_{\tilde{B}^{2}}\right) \\
\Delta P_{9}= & \frac{5 M^{11} \nu^{2}}{6864\left(\gamma^{2}-1\right) \Gamma}\left\{6 4 \left[\left(4800 \gamma^{8}+77520 \gamma^{6}-74888 \gamma^{4}+17707 \gamma^{2}+1888\right) \lambda_{\tilde{E}^{2}}\right.\right. \\
& \left.+16\left(300 \gamma^{8}+4845 \gamma^{6}-5467 \gamma^{4}+347 \gamma^{2}-25\right) \lambda_{\tilde{B}^{2}}\right]-9009\left(2 \gamma^{2}-1\right) \\
& \times \Gamma\left[\left(21 \gamma^{6}+385 \gamma^{4}-305 \gamma^{2}+91\right) \lambda_{\tilde{E}^{2}}+7\left(3 \gamma^{6}+55 \gamma^{4}-55 \gamma^{2}-3\right) \lambda_{\tilde{B}^{2}}\right] \\
& +\frac{16 \nu}{\left(\gamma^{2}-1\right)^{2}}\left[\left(27456 \gamma^{13}-19200 \gamma^{12}+205920 \gamma^{11}-271680 \gamma^{10}-1468896 \gamma^{9}\right.\right. \\
& +900512 \gamma^{8}+21724560 \gamma^{7}-980012 \gamma^{6}+580453302 \gamma^{5}+433656 \gamma^{4} \\
& \left.+837773079 \gamma^{3}-55724 \gamma^{2}+155291994 \gamma-7552\right) \kappa_{\tilde{E}^{2}} \\
& +\left(27456 \gamma^{13}-19200 \gamma^{12}+205920 \gamma^{11}-271680 \gamma^{10}-1589016 \gamma^{9}+950848 \gamma^{8}\right. \\
& +22048884 \gamma^{7}-1032064 \gamma^{6}+579540390 \gamma^{5}+395904 \gamma^{4}+826613931 \gamma^{3} \\
& \left.-25408 \gamma^{2}+148331040 \gamma+1600\right) \kappa_{\tilde{B}^{2}}+90090\left(\left(7292 \gamma^{6}+19644 \gamma^{4}+8141 \gamma^{2}+310\right) \kappa_{\tilde{E}^{2}}\right. \\
& \left.\left.\left.+\left(7292 \gamma^{6}+19484 \gamma^{4}+7905 \gamma^{2}+288\right) \kappa_{\tilde{B}^{2}}\right) a_{\mathrm{sh}}(\gamma)\right]\right\}
\end{aligned}
$$

Following the steps described in [59] we obtain the PM coefficients of the Hamiltonian displayed in the main text, where the $D_{i, j}$ polynomials are given by: 


$$
\begin{aligned}
& D_{7,1}=-8\left(224 \gamma^{9}-320 \gamma^{8}-728 \gamma^{7}+704 \gamma^{6}+5628 \gamma^{5}-528 \gamma^{4}+65982 \gamma^{3}+154 \gamma^{2}+28329 \gamma-10\right) \text {, } \\
& D_{7,2}=-8\left(224 \gamma^{9}-320 \gamma^{8}-728 \gamma^{7}+704 \gamma^{6}+5488 \gamma^{5}-444 \gamma^{4}+66262 \gamma^{3}+56 \gamma^{2}+28084 \gamma+4\right) \text {, } \\
& D_{7,3}=1680\left(440 \gamma^{4}+474 \gamma^{2}+33\right) \text {, } \\
& D_{7,4}=1680\left(440 \gamma^{4}+474 \gamma^{2}+32\right) \text {, } \\
& D_{7,5}=-16\left(160 \gamma^{6}-192 \gamma^{4}+72 \gamma^{2}-5\right) \text {, } \\
& D_{7,6}=-16\left(160 \gamma^{6}-192 \gamma^{4}+30 \gamma^{2}+2\right) \text {, } \\
& D_{7,7}=7\left(700 \gamma^{8}-1110 \gamma^{6}+597 \gamma^{4}-96 \gamma^{2}-11\right), \\
& D_{7,8}=35\left(140 \gamma^{6}-82 \gamma^{4}-\gamma^{2}-1\right) \text {, } \\
& D_{7,9}=7\left(1750 \gamma^{8}-1050 \gamma^{7}-2785 \gamma^{6}+1655 \gamma^{5}+1436 \gamma^{4}-952 \gamma^{3}-181 \gamma^{2}+203 \gamma-44\right) \text {, } \\
& D_{7,10}=35\left(350 \gamma^{6}-210 \gamma^{5}-207 \gamma^{4}+121 \gamma^{3}-3 \gamma^{2}+\gamma-4\right) \text {, } \\
& D_{7,11}=14\left(490 \gamma^{7}-280 \gamma^{6}-895 \gamma^{5}+380 \gamma^{4}+536 \gamma^{3}-208 \gamma^{2}-115 \gamma+44\right) \text {, } \\
& D_{7,12}=70\left(98 \gamma^{5}-56 \gamma^{4}-81 \gamma^{3}+20 \gamma^{2}+7 \gamma+4\right) \text {, } \\
& D_{9,1}=-27456 \gamma^{13}+19200 \gamma^{12}-205920 \gamma^{11}+271680 \gamma^{10}+1468896 \gamma^{9}-900512 \gamma^{8} \\
& -21724560 \gamma^{7}+980012 \gamma^{6}-580453302 \gamma^{5}-433656 \gamma^{4}-837773079 \gamma^{3}+55724 \gamma^{2}-155291994 \gamma+7552, \\
& D_{9,2}=-27456 \gamma^{13}+19200 \gamma^{12}-205920 \gamma^{11}+271680 \gamma^{10}+1589016 \gamma^{9}-950848 \gamma^{8} \\
& -22048884 \gamma^{7}+1032064 \gamma^{6}-579540390 \gamma^{5}-395904 \gamma^{4}-826613931 \gamma^{3}+25408 \gamma^{2}-148331040 \gamma-1600, \\
& D_{9,3}=90090\left(7292 \gamma^{6}+19644 \gamma^{4}+8141 \gamma^{2}+310\right) \text {, } \\
& D_{9,4}=90090\left(7292 \gamma^{6}+19484 \gamma^{4}+7905 \gamma^{2}+288\right) \text {, } \\
& D_{9,5}=-4\left(4800 \gamma^{8}+77520 \gamma^{6}-74888 \gamma^{4}+17707 \gamma^{2}+1888\right) \text {, } \\
& D_{9,6}=-4\left(4800 \gamma^{8}+77520 \gamma^{6}-87472 \gamma^{4}+5552 \gamma^{2}-400\right) \text {, } \\
& D_{9,7}=588 \gamma^{10}+8694 \gamma^{8}-13695 \gamma^{6}+6881 \gamma^{4}-1033 \gamma^{2}-91 \text {, } \\
& D_{9,8}=588 \gamma^{8}+9282 \gamma^{6}-6013 \gamma^{4}+196 \gamma^{2}-21 \text {, } \\
& D_{9,9}=1470 \gamma^{10}-882 \gamma^{9}+20881 \gamma^{8}-13895 \gamma^{7}-33371 \gamma^{6}+21409 \gamma^{5}+16239 \gamma^{4} \\
& -11285 \gamma^{3}-1975 \gamma^{2}+2157 \gamma-364 \\
& D_{9,10}=7\left(210 \gamma^{8}-126 \gamma^{7}+3193 \gamma^{6}-2111 \gamma^{5}-2100 \gamma^{4}+1336 \gamma^{3}+53 \gamma^{2}-59 \gamma-12\right) \text {, } \\
& D_{9,11}=2\left(420 \gamma^{9}-210 \gamma^{8}+5656 \gamma^{7}-3745 \gamma^{6}-10464 \gamma^{5}+4975 \gamma^{4}+5928 \gamma^{3}-2435 \gamma^{2}-1156 \gamma+455\right) \text {, } \\
& D_{9,12}=14\left(60 \gamma^{7}-30 \gamma^{6}+868 \gamma^{5}-565 \gamma^{4}-764 \gamma^{3}+260 \gamma^{2}+92 \gamma+15\right) \text {. }
\end{aligned}
$$

[1] B. P. Abbott et al. (LIGO Scientific, Virgo Collaborations), Phys. Rev. X 9, 031040 (2019).

[2] R. Abbott et al. (LIGO Scientific, Virgo Collaborations), arXiv:1912.11716.

[3] B. P. Abbott et al. (LIGO Scientific, Virgo Collaborations), Phys. Rev. Lett. 119, 161101 (2017).

[4] A. Buonanno and B. Sathyaprakash, arXiv:1410.7832.

[5] R. A. Porto, Fortsch. Phys. 64, 723 (2016).
[6] R. A. Porto, arXiv:1703.06440.

[7] E. E. Flanagan and T. Hinderer, Phys. Rev. D 77, 021502 (2008).

[8] T. Dietrich, T. Hinderer, and A. Samajdar, arXiv:2004.02527.

[9] B. Abbott et al. (LIGO Scientific, Virgo Collaborations), Phys. Rev. Lett. 121, 161101 (2018).

[10] A. Arvanitaki, M. Baryakhtar, S. Dimopoulos, S. Dubovsky, and R. Lasenby, Phys. Rev. D 95, 043001 (2017). 
[11] D. Baumann, H. S. Chia, and R. A. Porto, Phys. Rev. D 99, 044001 (2019).

[12] D. Baumann, H. S. Chia, R. A. Porto, and J. Stout, Phys. Rev. D 101, 083019 (2020).

[13] W. D. Goldberger and I. Z. Rothstein, Phys. Rev. D 73, 104029 (2006).

[14] W. Goldberger and I. Rothstein, Phys. Rev. D 73, 104030 (2006).

[15] J. B. Gilmore and A. Ross, Phys. Rev. D 78, 124021 (2008).

[16] S. Foffa and R. Sturani, Phys. Rev. D 84, 044031 (2011).

[17] S. Foffa and R. Sturani, Phys. Rev. D 87, 064011 (2013).

[18] C. Galley, A. Leibovich, R. A. Porto, and A. Ross, Phys. Rev. D 93, 124010 (2016).

[19] S. Foffa, P. Mastrolia, R. Sturani, and C. Sturm, Phys. Rev. D 95, 104009 (2017).

[20] R. A. Porto and I. Rothstein, Phys. Rev. D 96, 024062 (2017).

[21] S. Foffa and R. Sturani, Phys. Rev. D 100, 024047 (2019).

[22] S. Foffa, R. A. Porto, I. Rothstein, and R. Sturani, Phys. Rev. D 100, 024048 (2019).

[23] S. Foffa, P. Mastrolia, R. Sturani, C. Sturm, and W. J. T. Bobadilla, Phys. Rev. Lett. 122, 241605 (2019).

[24] J. Blümlein, A. Maier, and P. Marquard, Phys. Lett. B 800, 135100 (2020).

[25] J. Blümlein, A. Maier, P. Marquard, and G. Schäfer, Phys. Lett. B 807, 135496 (2020).

[26] W. D. Goldberger and A. Ross, Phys. Rev. D 81, 124015 (2010).

[27] C. R. Galley and A. K. Leibovich, Phys. Rev. D 86, 044029 (2012).

[28] A. K. Leibovich, N. T. Maia, I. Z. Rothstein, and Z. Yang, Phys. Rev. D 101, 084058 (2020).

[29] R. A. Porto, Phys. Rev. D 73, 104031 (2006).

[30] R. A. Porto and I. Rothstein, Phys. Rev. Lett. 97, 021101 (2006).

[31] R. A. Porto and I. Z. Rothstein, arXiv:0712.2032.

[32] R. A. Porto, Phys. Rev. D 77, 064026 (2008).

[33] R. A. Porto and I. Z. Rothstein, Phys. Rev. D 78, 044012 (2008).

[34] R. A. Porto and I. Z. Rothstein, Phys. Rev. D 78, 044013 (2008).

[35] R. A. Porto, Classical Quantum Gravity 27, 205001 (2010).

[36] R. A. Porto, A. Ross, and I. Z. Rothstein, J. Cosmol. Astropart. Phys. 03 (2011) 009.

[37] R. A. Porto, A. Ross, and I. Z. Rothstein, J. Cosmol. Astropart. Phys. 09 (2012) 028.

[38] N. T. Maia, C. R. Galley, A. K. Leibovich, and R. A. Porto, Phys. Rev. D 96, 084064 (2017).

[39] N. T. Maia, C. R. Galley, A. K. Leibovich, and R. A. Porto, Phys. Rev. D 96, 084065 (2017).

[40] M. Levi and J. Steinhoff, arXiv:1607.04252.

[41] M. Levi, A. J. Mcleod, and M. Von Hippel, arXiv:2003 .02827 .

[42] M. Levi, A. J. Mcleod, and M. Von Hippel, arXiv:2003 .07890 .

[43] W. D. Goldberger, arXiv:hep-ph/0701129.

[44] S. Foffa and R. Sturani, Classical Quantum Gravity 31, 043001 (2014).

[45] I. Rothstein, Gen. Relativ. Gravit. 46, 1726 (2014).
[46] V. Cardoso and R. A. Porto, Gen. Relativ. Gravit. 46, 1682 (2014).

[47] R. A. Porto, Phys. Rep. 633, 1 (2016).

[48] Q. Henry, G. Faye, and L. Blanchet, Phys. Rev. D 101, 064047 (2020).

[49] D. Neill and I. Z. Rothstein, Nucl. Phys. B877, 177 (2013).

[50] C. Cheung, I. Z. Rothstein, and M. P. Solon, Phys. Rev. Lett. 121, 251101 (2018).

[51] N. E. J. Bjerrum-Bohr, P. H. Damgaard, G. Festuccia, L. Planté, and P. Vanhove, Phys. Rev. Lett. 121, 171601 (2018).

[52] Z. Bern, C. Cheung, R. Roiban, C.-H. Shen, M. P. Solon, and M. Zeng, Phys. Rev. Lett. 122, 201603 (2019).

[53] Z. Bern, C. Cheung, R. Roiban, C.-H. Shen, M. P. Solon, and M. Zeng, J. High Energy Phys. 10 (2019) 206.

[54] A. Cristofoli, N. E. J. Bjerrum-Bohr, P. H. Damgaard, and P. Vanhove, Phys. Rev. D 100, 084040 (2019).

[55] D. A. Kosower, B. Maybee, and D. O'Connell, J. High Energy Phys. 02 (2019) 137.

[56] C. Cheung and M. P. Solon, J. High Energy Phys. 06 (2020) 144.

[57] Z. Bern, A. Luna, R. Roiban, C.-H. Shen, and M. Zeng, arXiv:2005.03071.

[58] J. Parra-Martinez, M. S. Ruf, and M. Zeng, arXiv:2005 .04236 .

[59] G. Kälin and R. A. Porto, J. High Energy Phys. 01 (2020) 072.

[60] G. Kälin and R. A. Porto, J. High Energy Phys. 02 (2020) 120.

[61] T. Damour, Phys. Rev. D 94, 104015 (2016).

[62] T. Damour, Phys. Rev. D 97, 044038 (2018).

[63] D. Bini, T. Damour, and A. Geralico, Phys. Rev. Lett. 123, 231104 (2019).

[64] T. Damour, Phys. Rev. D 102, 024060 (2020).

[65] D. Bini, T. Damour, and A. Geralico, Phys. Rev. D 101, 044039 (2020).

[66] G. Kälin and R. A. Porto, arXiv:2006.01184.

[67] G. Kälin, Z. Liu, and R. A. Porto, arXiv:2007.04977.

[68] K. Haddad and A. Helset, arXiv:2008.04920.

[69] C. Cheung and M. P. Solon, Phys. Rev. Lett. 125, 191601 (2020).

[70] T. Binnington and E. Poisson, Phys. Rev. D 80, 084018 (2009).

[71] T. Damour and A. Nagar, Phys. Rev. D 80, 084035 (2009).

[72] B. Kol and M. Smolkin, J. High Energy Phys. 02 (2012) 010.

[73] V. Cardoso, M. Kimura, A. Maselli, and L. Senatore, Phys. Rev. Lett. 121, 251105 (2018).

[74] A. Brandhuber and G. Travaglini, J. High Energy Phys. 01 (2020) 010.

[75] M. A. Huber, A. Brandhuber, S. De Angelis, and G. Travaglini, Phys. Rev. D 102, 046014 (2020).

[76] A. Le Tiec, L. Blanchet, and B. F. Whiting, Phys. Rev. D 85, 064039 (2012).

[77] B. Abbott et al. (LIGO Scientific, Virgo Collaborations), Astrophys. J. Lett. 892, L3 (2020).

[78] R. Abbott et al. (LIGO Scientific, Virgo Collaborations), Astrophys. J. 896, L44 (2020).

[79] A. Le Tiec and M. Casals, arXiv:2007.00214.

[80] J. M. Martín-García et al., www.xact.es (2019). 